\title{
Outcomes of 556 consecutive patients with stage I-III colon cancer managed in a single center over 10 years
}

\author{
Eleni Xenophontos ${ }^{a}$, Ifigenia Konstantinou ${ }^{a}$, Panteleimon Kountourakis ${ }^{a}$, Vassilios Vassiliou ${ }^{a}$, \\ Petros Polyviou ${ }^{a}$, Paris Vogazianos ${ }^{b}$, Demetris Papamichael ${ }^{a}$
}

Bank of Cyprus Oncology Center; European University of Cyprus

\section{Abstract}

Background The Bank of Cyprus Oncology Center is the largest cancer center in Cyprus, providing standalone oncology services to a population of just under a million.

Methods The aim of the study was to calculate disease-free survival (DFS) and overall survival (OS) for consecutive patients with stage I-III colon cancer over a 10-year period, by collecting retrospective data from patients' medical charts.

Results We identified 556 patients with a median age at diagnosis of 67 (range 18-88). The majority of them were male $(60 \%)$. Just over half of stage II patients received chemotherapy: capecitabine (44\%) and FOLFOX/CapeOx (7\%). Treatment administered in stage III was as follows: CapeOx (48\%); FOLFOX (28\%); capecitabine (12\%); 5-fluorouracil (4\%); and 8\% received no treatment. DFS at 5 years was: stage I 90\%; stage II 85\%; and stage III 69\%. Cancer-specific OS at 5 years was: stage I 94\%; stage II 93\%; and stage III 81\%. Favorable outcomes were also maintained at 10 years (stage I 94\%; stage II 84\%; and stage III 70\%). On multivariate analysis, only stage was statistically significant as a prognostic factor, whereas high-risk features ( $\mathrm{pT} 4 \pm \mathrm{pN} 2)$, disease location (right vs. left), and age $>70$ years old did not reach statistical significance.

Conclusions Despite our country's fragmented healthcare system, with multiple referring surgeons from the private and public sectors, the outcomes achieved were highly consistent with those published in the international literature. This can be attributed to optimal multidisciplinary management and follow-up care.

Keywords Colon cancer, early stage, single center

Ann Gastroenterol 2021; 34 (2): 1-5

\section{Introduction}

Colon cancer (CC) is the third most common cancer worldwide and the second leading cause of cancer-related death after lung cancer [1]. By 2040 the incidence and mortality from CC are anticipated to increase by $72 \%$ and $82 \%$, respectively [1]. Half of the new CC cases occur in developed

${ }^{a}$ Bank of Cyprus Oncology Center (Eleni Xenophontos, Ifigenia Konstantinou, Panteleimon Kountourakis, Vassilios Vassiliou, Petros

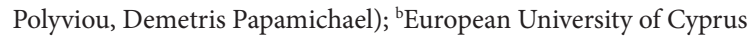
(Paris Vogazianos)

\section{Conflict of Interest: None}

Correspondence to: Eleni Xenophontos, Bank of Cyprus Oncology Center, Acropoleos 32, 2006, Nicosia, Cyprus,

e-mail: eleni.xenophontos@bococ.org.cy

Received 15 October 2020; accepted 15 December 2020; published online 26 February 2021

DOI: https://doi.org/10.20524/aog.2021.0603 countries and tumors are expected to plateau or decrease during the next few years, whilst developing countries are likely to witness an increase [1]. Survival outcomes from CC differ between developed and developing countries. According to Eurostat, Cyprus recorded the lowest share of deaths from CC among European Union countries in 2018 [2].

CC incidence and mortality are substantially higher in male than in female patients (30\% and $40 \%$ respectively) [3]. The reasons for this sex disparity are still unknown. Advanced age is another risk factor for sporadic CC. The median age at diagnosis is 68 years in men and 72 years in women and it is more prevalent between the ages 65 and 74 years old [1]. Leftsided CCs are more prevalent than right-sided ones, although a gradual increase has been recorded in right-sided CCs [4]. Based on the heterogeneity of epidemiological, histological and clinical outcomes between proximal and distal CCs, it has now become clearer that right-sided CCs have different molecular and genomic characteristics compared to leftsided CCs [5]. Proximal tumors tend to affect mostly older females, have mucinous and signet-ring histology, are poorly differentiated, metastasize to the peritoneum, are more 
likely to have characteristics of microsatellite instability-high (MSI-H)/mismatch repair (MMR) deficiency, and carry BRAF mutations.

In early stages (I-II), surgery alone can be curative in more than $85 \%$ of patients [6]. The potential benefit of adjuvant chemotherapy in stage II CC is still considered controversial. The QUASAR trial showed that adjuvant 5-fluorouracil (5-FU) with folinic acid was associated with an absolute survival benefit of only $3.6 \%$ [7]. However, the absolute improvement is small; current guidelines suggest considering adjuvant chemotherapy for a selected group of patients with high-risk features after balancing potential side-effects and patients' wishes [8]. Prognostic factors that inversely affect survival are: poor differentiation, less than 12 lymph nodes harvested in the surgical specimen, perineural or perivascular invasion, obstruction or perforation at initial presentation and pT4 tumors. The small subset of stage II patients (10-15\%) with MSI-H/MMR deficiency phenotype have very low risk of recurrence and do not seem to gain any benefit from additional treatment with 5-FU $[9,10]$.

Patients presenting with metastases to regional lymphnodes have a 5-year overall survival (OS) ranging between 30 and $50 \%$ with surgical resection alone. Early trials from the $90 \mathrm{~s}$ showed that treatment with 5-FU plus leucovorin for 6 months decreased the risk of death by $10-15 \%$ [11]. The MOSAIC trial investigated the role of oxaliplatin-based chemotherapy in patients with stage III CC and clearly showed that it offered an additional 4-6\% benefit in disease-free survival (DFS) and OS compared to 5-FU monotherapy [12]. This became the standard of care for this group of patients. Oxaliplatin combined with infusional 5-FU (FOLFOX4), or with capecitabine tablets (XELOX), are the most preferred regimens in this setting [8]. In 2018, the collaborators on the IDEA trial showed that 3 months of CAPOX is not inferior to 6 months of CAPOX in the subgroup analysis of patients with average risk characteristics. However, patients with high-risk features (pT4 and/or pN2) should be considered for the 6-month regimen [13]. Patients unable to receive oxaliplatin are candidates for infusional 5-FU or capecitabine monotherapy [14].

\section{Patients and methods}

The Bank of Cyprus Oncology Center (BOCOC) was established in 1998 and is the largest oncology center in the country, providing tertiary oncology services to a population of just under a million. This was a retrospective data analysis of patients who had been diagnosed with non-metastatic colon adenocarcinoma (stages I-III) between 1 January 2007 and 31 December 2017, and were managed in our center. Information was retrieved from the Cyprus Cancer Registry.

Epidemiological data were extracted from medical charts and electronic files. Patients who came only once looking for a second opinion (95 cases: stage I 22; stage II 39; and stage III 34) and those with missing date sequences (36 cases) were excluded from the analysis. All patients were censored from the time of diagnosis until 31 January 2017, or until death, whichever occurred first. Patients' status at the end of the study was confirmed by the national data registry archives.

Right CC was defined as a tumor located from the cecum up to the proximal $2 / 3$ of the transverse colon, and left CC tumors were those detected beyond the splenic flexure. In the small number of cases with multiple tumors $(n=9)$, we used the data for the tumor with the more advanced stage. Stage was defined according to the TNM $7^{\text {th }}$ edition. Descriptive statistics were used to present the clinical characteristics of the population.

Our primary endpoints were DFS and OS at 5 and 10 years after diagnosis. Date of diagnosis was defined as the date of the first biopsy. DFS was defined as the time between initial diagnosis and objective disease progression or death or study close date. OS was defined as the time between diagnosis and death from any cause, while cancer-specific OS (CSOS) was the time between diagnosis and death from CC. Progression was defined according to the response evaluation criteria in solid tumors (RECIST) rules. DFS, OS and CSOS were calculated by the Kaplan-Meier method, and the log-rank test with a $95 \%$ confidence interval (CI) was performed to make comparisons between the survival and progression curves.

A secondary multivariate analysis was performed to examine the associations of age, high risk factors (T4 and/or N2) and tumor side (in stage III patients) with survival. The Cox proportional hazard regression model was used in this case. We also sought to investigate the number of referring surgeons during this time period.

\section{Results}

The study cohort comprised 556 consecutive patients with stage I, II or III CC registered at BOCOC between 1 January 2007 and 31 December 2017. The majority were male and their median age at diagnosis was 67 years, with a range of 18-88 years (Table 1). The sigmoid colon was the most common site of disease ( $40 \%$ of the cases) and the cecum was the second (22\%). The majority of patients were diagnosed at an early stage ( $42 \%$ at stage II and $17 \%$ at stage I), while $39 \%$ had a locally advanced disease.

All patients who presented with stage I disease were followed-up for 5 years, with physical examination and serial measurements of carcinoembryonic antigen (CEA) and cancer antigen 19-9 every 3 months for the first 3 years and every 6 months thereafter, until the completion of 5 years. Colonoscopy was advised at 1 year from diagnosis and at year 4 . After 5 years of follow up, patients were discharged back to their general practitioner. Molecular biomarkers were available for 63 (11\%) patients and were as follows: KRASmut 34; NRASmut 1; BRAFmut 3; and MSI-H 25. High-risk features that merited adjuvant chemotherapy in stage II disease (T4, Grade 3, <12 lymph nodes harvested during the surgery, perforation or obstruction, perineural or lymphovascular invasion, R1 excision) were present in 177 cases (73\%) (Table 2). Some patients had more than 1 risk factor. Almost half of stage II patients did not receive any adjuvant treatment (49\%), while the rest had capecitabine for 6 months. Combination chemotherapy (CapeOx/FOLFOX) 
was administered to a minority (7\%) of patients, who had more than 1 risk factor. Patients with node-positive disease at diagnosis received combination chemotherapy for 6 months (48\% CapeOx and 29\% FOLFOX); of the remainder, 15\% had monotherapy with fluoropyrimidine analogs (12\% capecitabine and $3 \% 5-\mathrm{FU}$ ) and $8 \%$ received no treatment.

In patients diagnosed with stage I CC the risk of relapse at 5 and 10 years was $10 \%$ (hazard ratio [HR] 0.90, 95\%CI 0.84-

$\underline{\text { Table } 1 \text { Demographic and clinical characteristics }}$

\begin{tabular}{lc}
\hline Characteristics & Value \\
\hline Sex & \\
Male & $332(60 \%)$ \\
Female & $224(40 \%)$ \\
Ratio & $3: 2$ \\
Age (years) & \\
Mean & 65 \\
Median & 66.76 \\
Range & $18-88$ \\
Site & \\
Cecum & 121 \\
Ascending & 69 \\
Transverse & 85 \\
Descending & 43 \\
Sigmoid & 229 \\
Multifocal & 556 \\
Stages & $918(39 \%)$ \\
Stage I & 12 \\
Stage II & 182 \\
Stage II A (T3N0) & 24 \\
Stage II B (T4N0) & \\
Stage III & $96(17 \%)$ \\
Stage III A (T1-2N1, T1N2a) III B (T3-4N1, T2-3N2b, T1-2N2b) & \\
Total & \\
\hline & \\
\hline
\end{tabular}

Table 2 High-risk features in stage II colon cancer patients

\begin{tabular}{lc}
\hline Risk Factors & Number of patients \\
\hline T4 & 46 \\
Grade 3 & 18 \\
Lymph nodes harvested during surgery $<12$ & 110 \\
$\begin{array}{l}\text { Lymphovascular invasion or perineural } \\
\text { invasion }\end{array}$ & Not available \\
R1 & 1 \\
Perforation/obstruction & 2 \\
\hline
\end{tabular}

0.96 , at both 5 and 10 years); in patients with stage II it was $15 \%$ at 5 years (HR $0.85,95 \%$ CI $0.81-0.90)$ and $18 \%$ at 10 years (HR $0.82,95 \%$ CI 0.76-0.88); while in patients with stage III disease it was $31 \%$ at 5 years and increased by $5 \%$ at 10 years (HR 0.69 , 95\%CI $0.62-0.75$ and HR 0.64, 95\%CI 0.56-0.72) (Fig. 1). The risk of death for stage I was $12 \%$ (HR $0.88,95 \%$ CI $0.80-0.95$ ), for stage II $15 \%$ (HR $0.85,95 \%$ CI $0.80-0.90$ ), and for stage III 25\% (HR 0.75, 95\%CI 0.67-0.81) (Fig. 2). The risk of death at 10 years: stage I $16 \%$ (HR 0.84, 95\%CI 0.75-0.94), stage II 36\% (HR 0.64, 95\%CI 0.53-0.76), and stage III 39\% (HR 0.61, 95\%CI 0.51-0.71). All results were statistically significant $(\mathrm{P}<0.05)$. By excluding deaths from other causes, CSOS showed that the chance of being alive at 5 years for stage I was $94 \%$ (HR 0.94, 95\%CI 0.88-1), for stage II 93\% (HR 0.93, 95\%CI 0.89-0.96), and for stage III $81 \%$ (HR 0.81, 95\%CI 0.75-0.87) (Fig. 3). At 10 years CSOS values were as follows: stage I 94\% (HR 0.94, 95\%CI $0.88-1$ ), stage II $84 \%$ (HR $0.84,95 \%$ CI $0.78-0.91$ ), and stage III 70\% (HR 0.70, 95\%CI 0.61-0.80).

The vast majority of relapses occurred between the first and fourth years from diagnosis (28 at year 1, 47 at year 2, 19 at year 3, and 11 at year 4, irrespective of stage) (Fig. 4). We also encountered

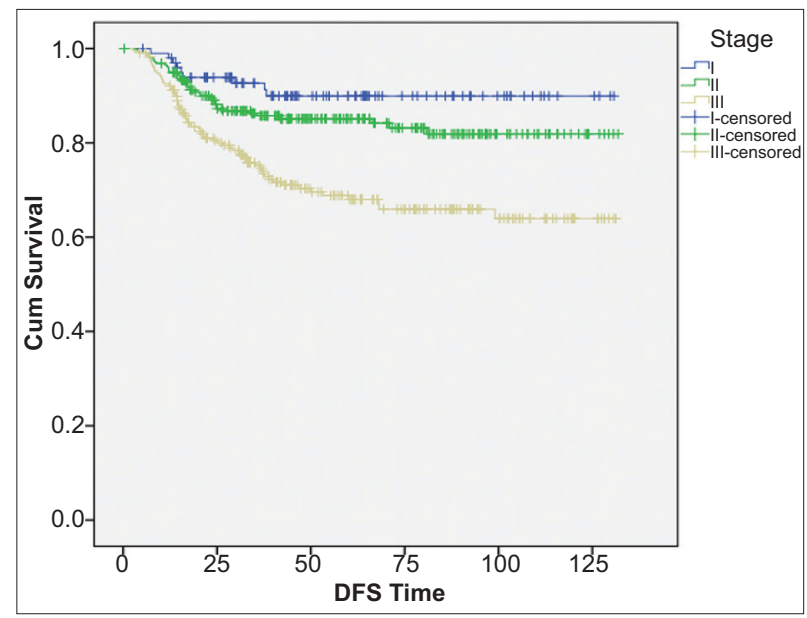

Figure 1 Disease-free survival (DFS) curves for stages I, II and III CI, confidence interval

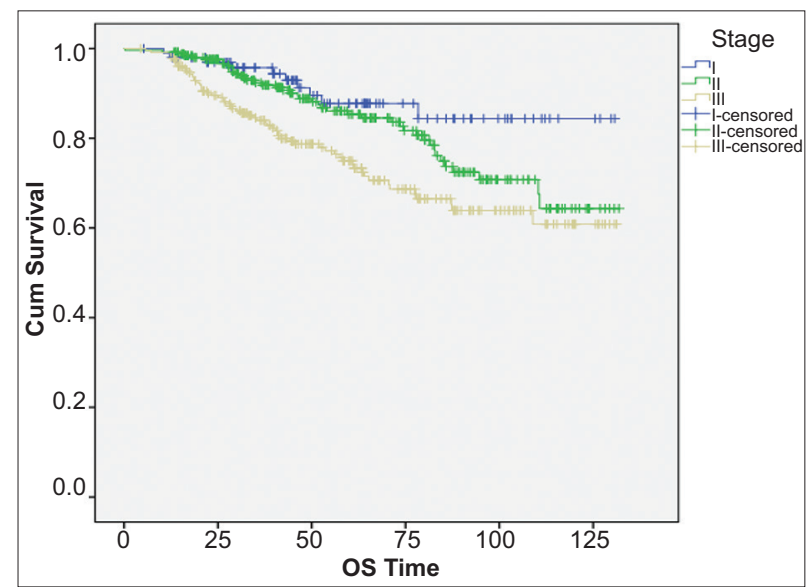

Figure 2 Overall survival (OS) curves for stages I, II and III CI, confidence interval 


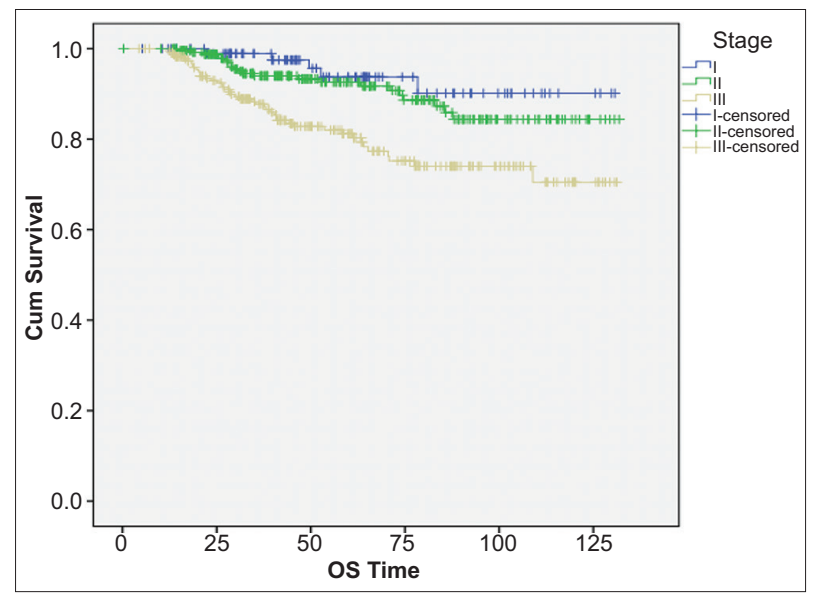

Figure 3 Cancer-specific overall survival (CSOS) for stages I, II and III

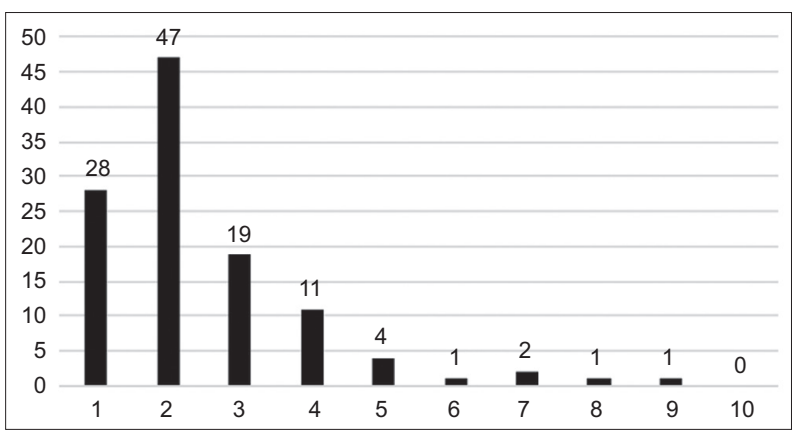

Figure 4 Relapses during years of follow up. Vertical axis shows number of relapses and horizontal axis years of follow up

relapses between years 5 and 9 of the initial diagnosis (in total 9), but none at year 10. Patients with stage III disease experienced the most relapses $(n=67)$, which occurred during years 1-9. Patients with stage I disease had far fewer relapses $(n=9)$, which happened during years 1-4. At stage II, 38 relapses were censored and occurred during years 1-7. Of those who experienced a relapse the majority were treated by palliative chemotherapy (39\%), 15\% had a metastasectomy, $11 \%$ had perioperative chemotherapy, $11 \%$ had radiofrequency ablation (RFA), 3\% palliative radiotherapy (RT), $16 \%$ were treated by best supportive care, and the remaining $5 \%$ had a combination of metastasectomy-RFA-chemotherapy and metastasectomy-RT-chemotherapy.

Age ( $\leq 70$ and $>70$ years) was not associated with any survival difference in our study. Older patients with stage II CC ( $>70$ years) achieved the same DFS, irrespectively of whether they received adjuvant therapy or not $(83.3 \%$ vs. $80.8 \%, \mathrm{P}=0.950)$; however, they had better OS if they had a treatment (91\% vs. $88.5 \%$, $\mathrm{P}=0.931)$. Older patients with stage III CC exhibited poorer DFS ( $66.7 \%$ vs. $56.3 \%, \mathrm{P}=0.431)$ and $\mathrm{OS}(75.4 \%$ vs. $68.8 \%, \mathrm{P}=0.460)$ if not treated, but again the results were statistically insignificant. The presence of high-risk features (T4 $\pm \mathrm{N} 2$ disease) in stage III patients conferred a poorer prognosis (DFS $69.2 \%$ vs. $72.5 \%$, $\mathrm{P}=0.455$; and $\mathrm{OS} 76.1 \%$ vs. $80.9 \%, \mathrm{P}=0.316$ ).

The association between tumor side (right vs. left) and survival in patients with stage III CC showed that patients with left-side CC did better than patients with right-sided CC. At 5 years, the risk of relapse for patients with proximal tumors was $38 \%$ (HR 0.62, 95\%CI 0.56-0.78) and for left 26\% (HR 0.74, 95\%CI 0.64-0.87) and became higher at 10 years: $43 \%$ (HR 0.57 , 95\%CI 0.46-0.68) for right-sided CC and 29\% (HR 0.71, 95\%CI $0.53-0.86)$ for left-sided CC. The HRs for OS at 5 years were 0.8 , 95\%CI 0.72-0.89 for left-sided CC and 0.61, 95\%CI 0.63-0.83 for right-sided CC, and at 10 years $0.73,95 \%$ CI $0.45-0.81$ vs. 0.45 $95 \% \mathrm{CI} 0.24-0.83$, respectively. These results should be interpreted with caution as they lacked statistical significance $(\mathrm{P}>0.05)$.

Patients were referred to us by multiple surgeons from all over the island (105 surgeons) and a minority (10 cases) by surgeons from Greece, the United Kingdom and Germany (6 cases). However, the majority were referred by surgical teams: 2 from the public sector ( 127 cases) and 2 from the private sector (115 cases).

\section{Discussion}

CC is a major health problem, accounting for more than 860,000 deaths worldwide in 2018 [1]. There is a discrepancy between developed and developing countries regarding incidence and survival, which underlines the geographic inequality and disparity. The risk of CC increases with age, although the current trends suggest an increase in younger ages and a decrease in individuals $>50$ years in developed countries [15]. The median age for CC in men is 68 years and in women 72 years [16]. In this study the median age was 67 years. This observation may reflect the earlier detection of CC by screening modalities, or perhaps patients seeking oncological help earlier. In addition, the incidence for men is higher than for women in this and other studies (male/female ratio: 3/2) for reasons not fully understood.

Stage at diagnosis has been the most important prognostic factor. The 5-year OS was $88 \%$ and $85 \%$ for stages I and II, respectively, and $75 \%$ for stage III. These rates are comparable with survival data from high-income countries such as the USA (5-year OS $89.8 \%$ in localized disease and $71.1 \%$ in patients with locally advanced disease) [17]. When deaths from other causes were excluded, our rates were substantially improved: 94\%, $93 \%$ and $81 \%$ for stages I, II and III, respectively. The very good outcomes achieved in our oncology center are assumed to be the result of optimal follow up, multidisciplinary care and aggressive management where appropriate at the time of relapse.

Despite optimal management of early-stage CC, 30-50\% of patients will relapse and die from their cancer. Our results showed that one third of patients with locally advanced disease and $15 \%$ of those with stage II CC will relapse at 5 years. Analyzing data from the ACCENT database, Sargent et al found that for patients destined to relapse, $82 \%$ of those with stage III and $74 \%$ of those with stage II will do so within 3 years after treatment. Therefore, for these patients 3-year DFS outcomes are excellent predictors of 5-year survival [18]. Some studies searching for the optimal surveillance strategy have suggested that intensive follow up leads to a $7-13 \%$ survival benefit during the first 5 years after diagnosis [19]. European Society for Medical Oncology guidelines recommend intensive follow up with clinical examination and serial CEA measurements 
every 3-6 months for the first 3 years after diagnosis and then every 6 months for the next 2 years, colonoscopy at the first year after diagnosis and then every 3-5 years, and computed tomography scan of the chest and abdomen every 6-12 months after diagnosis for the first 3 years [20].

Stage migration is another important factor implicated in improved survival rates. Shi et al compared the outcomes of old studies (1978-1995) and newer studies (1997-2007) in the adjuvant setting of CC and concluded that the improved survival observed in the modern era is due to better care and diagnosis at the first stages and more therapeutic options at the time of recurrence [21]. Our results may also reflect this phenomenon.

In conclusion, we presented the 10-year survival data of 556 consecutive patients from a standalone oncology center in Cyprus. Despite our fragmented healthcare system with multiple referring doctors, favorable 10-year survival results were achieved.

\section{Summary Box}

\section{What is already known:}

- Colon cancer (CC) is the third most common cancer worldwide and the second leading cause of cancer related death. Its prevalence and mortality are expected to increase mostly to an age population

- Right-sided CCs exhibit different clinical, molecular and genomic characteristics when compared to left-sided CCs

- Stage migration is potentially explains good comes, especially in stage II disease

\section{What the new findings are:}

- Optimal multidisciplinary management and follow-up care are key of the favorable outcomes

- Survival data from a stand-alone oncology center showed favorable outcomes that were highly consistent with those published in the international literature

\section{References}

1. Bray F, Ferlay J, Soerjomataram I, Siegel RL, Torre LA, Jemal A. Global cancer statistics 2018: GLOBOCAN estimates of incidence and mortality worldwide for 36 cancers in 185 countries. CA Cancer J Clin 2018;68:394-424.

2. Eurostat Statistics Explained. Cancer statistics - specific cancers. 2020. Available from: https://ec.europa.eu/eurostat/ statistics-explained/index.php/Cancer_statistics_-_specific_ cancers\#Colorectal_cancer [Accessed 26 January 2021].

3. International Agency for Research on Cancer, World Health Organization. Estimated age-standardized incidence rates (World) in 2018, worldwide, males and females, all ages. 2020. Available from: https://gco.iarc.fr [Accessed 26 January 2021].
4. Jessup JM, McGinnis LS, Steele GD, Menck HR, Winchester DP. The national cancer data base report on colon cancer. Cancer 1996;78:918-926.

5. Lee MS, Menter DG, Kopetz S. Right versus left colon cancer biology: integrating the consensus molecular subtypes. I Natl Compr Canc Netw 2017;15:411-419.

6. Gunderson LL, Jessup JM, Sargent DJ, Greene FL, Stewart AK. Revised TN categorization for colon cancer based on national survival outcomes data. J Clin Oncol 2010;28:264-271.

7. Gray R, Barnwell J, McConkey C, Hills RK, Williams NS, Kerr DJ; Quasar Collaborative Group. Adjuvant chemotherapy versus observation in patients with colorectal cancer: a randomised study. Lancet 2007;370:2020-2029.

8. Van Cutsem E, Nordlinger B, Cervantes A; ESMO Guidelines Working Group. Advanced colorectal cancer: ESMO Clinical Practice Guidelines for treatment. Ann Oncol 2010;21 Suppl 5:v93-v97.

9. Sargent DJ, Marsoni S, Monges G, et al. Defective mismatch repair as a predictive marker for lack of efficacy of fluorouracil-based adjuvant therapy in colon cancer. J Clin Oncol 2010;28:3219-3226.

10. Roth AD, Tejpar S, Delorenzi M, et al. Prognostic role of KRAS and BRAF in stage II and III resected colon cancer: results of the translational study on the PETACC-3, EORTC 40993, SAKK 60-00 trial. J Clin Oncol 2010;28:466-474.

11. Haller DG, Catalano PJ, Macdonald JS, et al. Phase III study of fluorouracil, leucovorin, and levamisole in high-risk stage II and III colon cancer: final report of Intergroup 0089. J Clin Oncol 2005;23:8671-8678.

12. André T, Boni C, Mounedji-Boudiaf L, et al; Multicenter International Study of Oxaliplatin/5-Fluorouracil/Leucovorin in the Adjuvant Treatment of Colon Cancer (MOSAIC) Investigators. Oxaliplatin, fluorouracil, and leucovorin as adjuvant treatment for colon cancer. N Engl J Med 2004;350:2343-2351.

13. André T, Vernerey D, Mineur L, et al; for PRODIGE investigators, GERCOR, Fédération Française de Cancérologie Digestive, and UNICANCER. Three versus 6 months of oxaliplatin-based adjuvant chemotherapy for patients with stage III colon cancer: disease-free survival results from a randomized, open-label, International Duration Evaluation of Adjuvant (IDEA) France, phase III trial. J Clin Oncol 2018;36:1469-1477.

14. Twelves C, Wong A, Nowacki MP, et al. Capecitabine as adjuvant treatment for stage III colon cancer. N Engl J Med 2005;352:2696-2704.

15. Mariotto AB, Noone AM, Howlader N, et al. Cancer survival: an overview of measures, uses, and interpretation. J Natl Cancer Inst Monogr 2014;2014:145-186.

16. Siegel RL, Miller KD, Fedewa SA, et al. Colorectal cancer statistics, 2017. CA Cancer J Clin 2017;67:177-193

17. Maringe C, Walters S, Rachet B, et al; ICBP Module 1 Working Group. Stage at diagnosis and colorectal cancer survival in six highincome countries: a population-based study of patients diagnosed during 2000-2007. Acta Oncol 2013;52:919-932.

18. Sargent DJ, Patiyil S, Yothers G, et al; ACCENT Group. End points for colon cancer adjuvant trials: observations and recommendations based on individual patient data from 20,898 patients enrolled onto 18 randomized trials from the ACCENT Group. J Clin Oncol 2007;25:4569-4574.

19. Jeffery M, Hickey BE, Hider PN. Follow-up strategies for patients treated for non-metastatic colorectal cancer. Cochrane Database Syst Rev 2019;9:CD002200.

20. Argilés G, Tabernero J, Labianca R, et al; ESMO Guidelines Committee. Electronic address: clinicalguidelines@esmo.org. Localised colon cancer: ESMO Clinical Practice Guidelines for diagnosis, treatment and follow-up. Ann Oncol 2020;31:1291-1305.

21. Shi Q, Andre T, Grothey A, et al. Comparison of outcomes after fluorouracil-based adjuvant therapy for stages II and III colon cancer between 1978 to 1995 and 1996 to 2007: evidence of stage migration from the ACCENT database. J Clin Oncol 2013;31:3656-3663. 